\title{
Thermodynamic Assessment of the Fe-B System in the Ssol5 and User Databases
}

\author{
Mateusz Szymanski*, Viera Homolová**, Marcin Leonowicz* \\ *(Faculty of Materials Science and Engineering, Warsaw University of Technology, 141 Wolosk a Street, 02 -507 \\ Warsaw, Poland) \\ ** (Institute of Materials Research, Slovak Academy of Sciences, Watsonova 47, Kořice 04001 Slovakia)
}

\begin{abstract}
Thermodynamic assessment of Fe-B system, including phase diagram, Gibbs energy, enthalpy, heat capacity and activity, was performed in the ThermoCalc software ver. 4.1 (Sweden). Two databases were used: the commercial SSOL5 database for solid solutions (substitutional approach) and the USER made database based on work of T. Van Rompaey et al. (intersticial approach). Results obtained were compared with experimental data gathered from work of M. Van Ende et al. In low boron regime the curve of the Fe-B phase diagram is represented more reliable in the USER database. However, temperatures of the phase transformations are calculated with more accuracy in the SSOL5 database. For boron content higher than 0.3 mole fraction phase transformation temperatures are better assessed in the USER database, except for melting point of the $\mathrm{Fe}_{2} \mathrm{~B}$ phase. Gibbs energy, enthalpy and heat capacity of the $\mathrm{FeB}$ and the $\mathrm{Fe}_{2} \mathrm{~B}$ are difficult to evaluate because experimental data are spread and inaccurate. Activities of iron and boron in liquid $\mathrm{Fe}-\mathrm{B}$ alloy, calculated at selected temperatures, are almost identical for both databases. paramount importance.
\end{abstract}

Keywords: thermocalc

\section{INTRODUCTION}

Iron and Boron are important constituents of steels and magnetic materials. There is a need to simulate properties and behavior of these products. The milestone papers allowing for calculation of the $\mathrm{Nd}-\mathrm{Fe}-\mathrm{B}$ system were provided by Hallemans et al. in 1994 [1] and 1995 [2]. Not so long ago, a significant article was published by Van Ende et al. who provided a critical review of all theoretical and experimental data available for the $\mathrm{Nd}-\mathrm{Fe}-\mathrm{B}$ system and proposed improved parameters for calculations [3]. Very recently Zhou et al. investigated thermodynamic description of the $\mathrm{Nd}-\mathrm{Fe}-\mathrm{B}$ system including metastable phases $\mathrm{Fe}_{3} \mathrm{~B}, \mathrm{Fe}_{17} \mathrm{Nd}_{2} \mathrm{~B}$ and $\mathrm{Fe}_{23} \mathrm{Nd}_{2} \mathrm{~B}_{3}$ which have effect on magnetic properties of dual-nanoscale-phase nanocomposite magnets [4]. However, obtained phase diagrams cannot be easily compared because one must suspend stable phases from calculations, e.g. $\mathrm{Fe}_{2} \mathrm{~B}$ phase, in order to display metastable one, e.g. $\mathrm{Fe}_{3} \mathrm{~B}$ phase. In order to predict ternary system one has to properly assess binary one first [5]. Therefore, this work is focused on the Fe-B system which is calculated using different approaches. In the SSOL5 database iron and boron are recognized as substitutional solutions, while in the USER database boron is modelled as an interstitial element in the FCC and BCC solid solutions.

\section{EXPERIMENTAL}

Calculations were performed using ThermoCalc ver. 4.1 software equipped with two databases: commercial SSOL5 for solid solutions (substitutional approach) based on Hallemans et al. $[1,2]$ and USER made database with data from work of T. Van Rompaey [6]. Conditions used in calculations (reference states, temperatures etc.) were based on those used in Van Ende's et al. [3] thus allowing for easy comparison. Experimental data, gathered by various authors, were read from Van Ende's et al. paper using DataThief III software developed by B. Tummers [7].

\section{RESULTS AND DISCUSSION}

Boron-rich part of calculated Fe-B phase diagram is presented together with experimental data in Fig. 1. For boron content up to 0.3 mole fraction transformation temperatures are too high in USER database (e.g. difference in eutectic transformation at $0.16 \mathrm{~B}$ is higher by more than $20^{\circ} \mathrm{C}$ between the databases). In contrary, transformation temperatures for boron content more than 0.3 mole fraction are reduced in the USER database (e.g. melting point of $\mathrm{FeB}$ phase is almost $30^{\circ} \mathrm{C}$ lower comparing with the SSOL5) what fits better experimental data. Melting point of the $\mathrm{FeB}$ phase, calculated at $1651.1^{\circ} \mathrm{C}$ byVan Ende et al., is reduced in both databases: $1632.6^{\circ} \mathrm{C}$ in the SSOL5 and $1602.8^{\circ} \mathrm{C}$ in the USER. Unfortunately, experimental data for the $\mathrm{FeB}$ compound varies significantly and one has to choose among the results. 


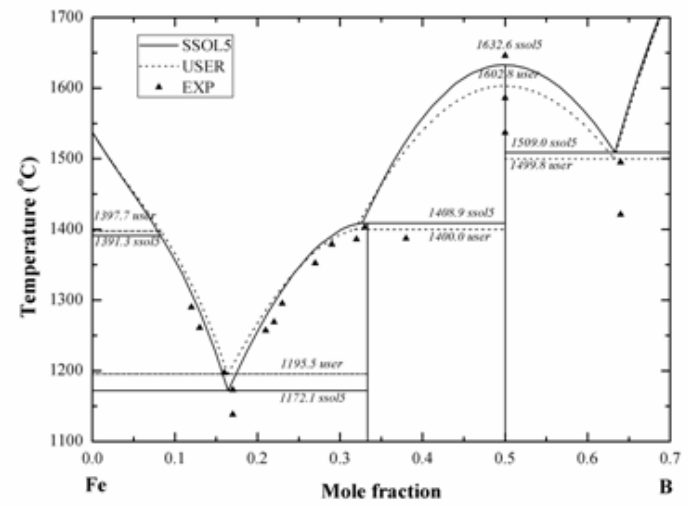

Fig. 1 Phase diagrams of the Fe-B system calculated in the SSOL5 (solid line) and the USER (dotted line) database with experimental data (triangles) obtained by various authors [3].

An iron-rich corner of calculated the $\mathrm{Fe}-\mathrm{B}$ phase diagram is presented together with experimental data in Fig. 2. A single phase region of the FCC phase is clearly visible only in the USER. Indeed, the FCC phase appears in the SSOL5 but has extremely narrow existence's range, making it practically invisible. This difference, including experimental results, should be considered in a favor of the USER database.

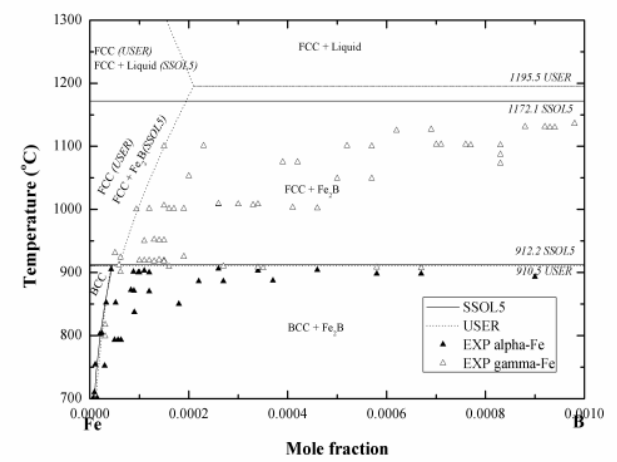

Fig. 2 Fe-rich corner of the Fe-B binary phase diagrams calculated in the SSOL5 (solid line) and the USER (dotted line) database with experimental data (triangles) obtained by various authors [3].

Despite the FCC phase exists in the SSOL5 database, it is virtually invisible in the left side of the diagram.

The heat capacity of $\mathrm{FeB}$ and $\mathrm{Fe}_{2} \mathrm{~B}$ versus temperature is presented together with experimental data in Fig. 3. Simulation for FeB calculated in the SSOL5 should be slightly elevated (around +5 $\mathrm{J} / \mathrm{mol}-\mathrm{K}$ ) at temperatures below $1300 \mathrm{~K}$ and slightly reduced at higher temperatures in order to cover the experimental data. These adjustments are actually what the USER database provides. Peaks denote Curie te mperature. Moreover, neither of the database fits experimental points measured above $1900 \mathrm{~K}$.
This part of curves should be elevated around +15 $\mathrm{J} / \mathrm{mol}-\mathrm{K}$.

The heat capacity of $\mathrm{Fe}_{2} \mathrm{~B}$ calculated in the SSOL5 covers experimental data well. Simulation performed in the USER lies too high and should be reduced. There are no experimental data for $\mathrm{Fe}_{2} \mathrm{~B}$ at temperatures above $1500 \mathrm{~K}$ so this part of curves cannot be verified.

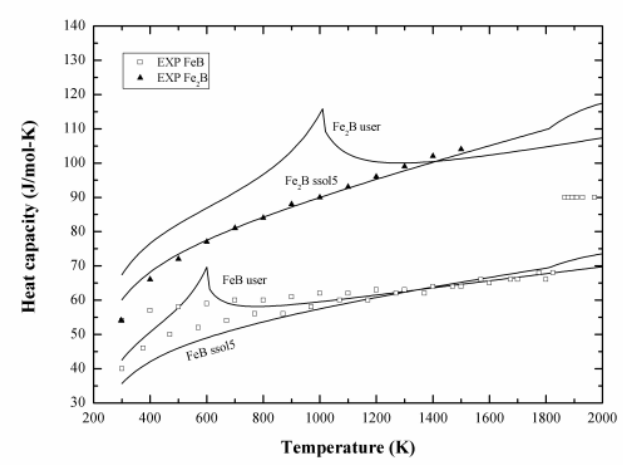

Fig. 3 Heat capacity of FeB and Fe2B calculated in the SSOL5 and the USER database using hfr(phase).t formula at standard reference states

(SER). Experimental data obtained by various authors (squares for $\mathrm{FeB}$ and asterisks for $\mathrm{Fe} 2 \mathrm{~B}$ ) [3].

Enthalpy of formation of $\mathrm{FeB}$ and $\mathrm{Fe}_{2} \mathrm{~B}$ calculated in the SSOL5 and the USER database is presented together with experimental data in Fig. 4. First of all, one should notice experimental points are spread and uncertain, hindering evaluation. Indeed, simulations obtained in the SSOL5 are closer to experimental data, both databases should be improved by elevating enthalpy values at temperatures above $1500 \mathrm{~K}$ for both $\mathrm{FeB}$ and $\mathrm{Fe}_{2} \mathrm{~B}$. Experimental points measured between $1000-1300 \mathrm{~K}$ do not fit any calculation at all including database improved by Van Ende.

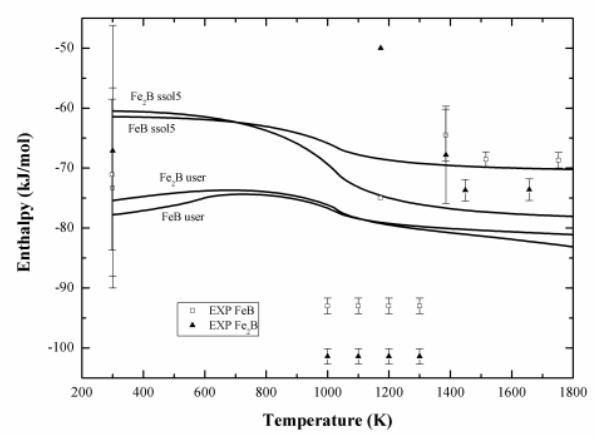

Fig. 4 Enthalpy of formation of $\mathrm{FeB}$ and $\mathrm{Fe}_{2} \mathrm{~B}$ calculated in the USER and the SSOL5 database with experimental data (squares for $\mathrm{FeB}$ and triangles for $\mathrm{Fe}_{2} \mathrm{~B}$ ) obtained by various authors [3]. The reference states are set as recommended: iron in $\mathrm{BCC}$ and boron in $\mathrm{B}_{\text {beta }}$ at default temperature. 
The Gibbs energy of $\mathrm{FeB}$ and $\mathrm{Fe}_{2} \mathrm{~B}$ calculated in the USER and the SSOL5 database is presented together with experimental data in Fig. 5. Experimental data are spread, inaccurate and there are no measuring points below $1000 \mathrm{~K}$. Calculations provided in both databases cover experimental data at temperatures between $1400-1700 \mathrm{~K}$ for $\mathrm{Fe}_{2} \mathrm{~B}$ but not for FeB. At temperatures between 1000-1300 K simulations for both databases should be reduced by around $-10 \mathrm{~kJ} / \mathrm{mol}$ in order to fit experimental points for both phases. Although. calculated curves are generally lower for the USER database value's reduction should be greater.

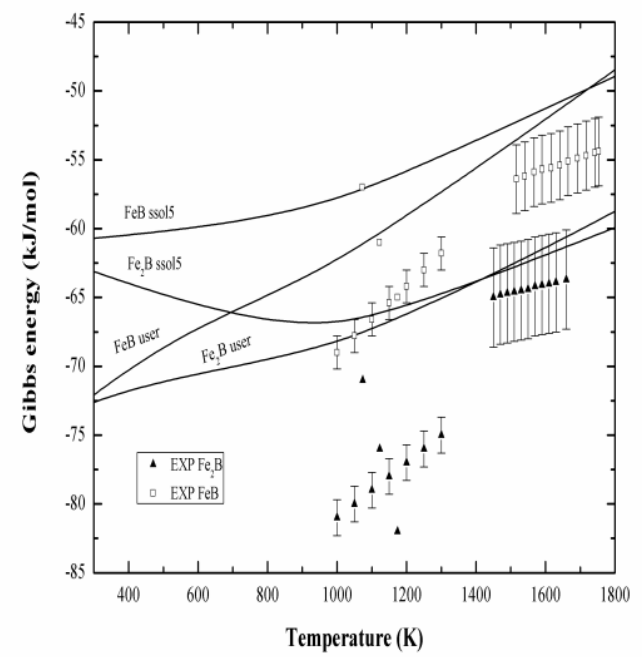

Fig. 5 Gibbs free energy of $\mathrm{FeB}$ and $\mathrm{Fe}_{2} \mathrm{~B}$ calculated in the SSOL5 and the USER database with experimental data (squares for $\mathrm{FeB}$ and triangles for $\mathrm{Fe}_{2} \mathrm{~B}$ ) obtained by various authors [3]. Reference states are: BCC for iron and BETA_RHOMBO_B for boron.

The activity of $\mathrm{Fe}$ and $\mathrm{B}$ in liquid $\mathrm{Fe}-\mathrm{B}$ alloy calculated at selected temperatures is presented with experimental data in Fig. 6-8 for the SSOL5 and in Fig. 9-11 for the USER database, respectively. One could see the activities of $\mathrm{Fe}$ and $\mathrm{B}$ are calculated almost identically in the two databases. Activity of Fe calculated in the USER database for around 0.5 boron content is slightly elevated at lower temperature $(1673 \mathrm{~K})$ and slightly reduced at higher temperatures $(1823 \mathrm{~K}$ and 1873 $\mathrm{K})$. The iron's activity curve at $1923 \mathrm{~K}$ seems to be the same for both databases. On the other hand, activity of $B$ calculated in the USER database is slightly elevated at higher temperatures $(1773 \mathrm{~K}$ and $1823 \mathrm{~K})$ and slightly reduced at lower temperature (1673 K).

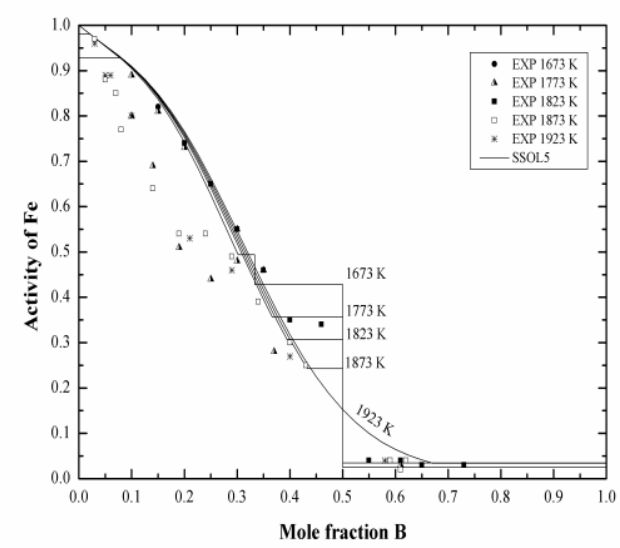

Fig. 6 Activ ity of Fe in liqu id Fe-B alloy calculated at selected temperatures in the SSOL5 database with experimental data [3].

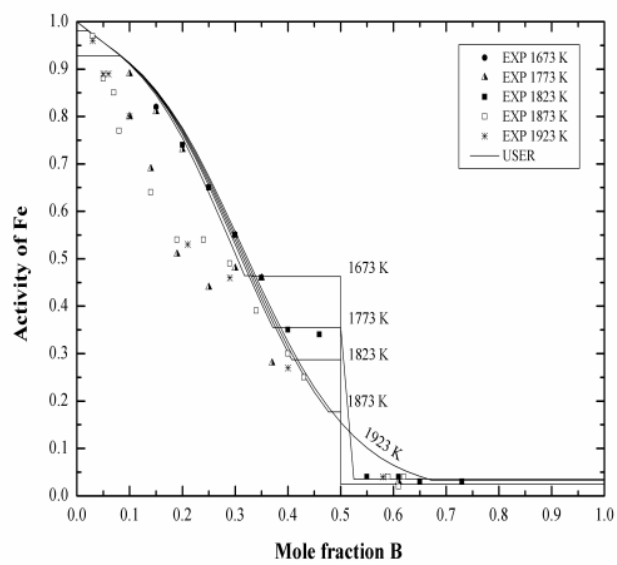

Fig. 7 Activity of Fe in liqu id Fe-B alloy calculated at selected temperatures in the USER database with experimental data [3].

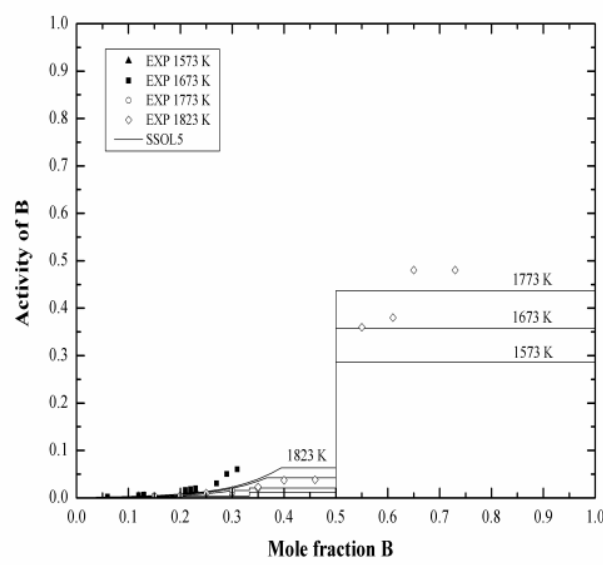

Fig. 8 Activity of B in liquid Fe-B alloy calculated at selected temperatures in the SSOL5 database with experimental data [3]. 


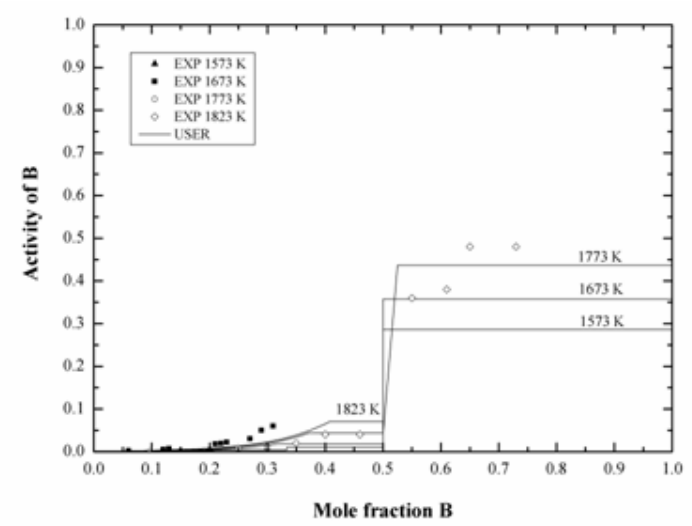

Fig. 9 Activity of B in liquid Fe-B alloy calculated at selected temperatures in the USER database with experimental data [3].

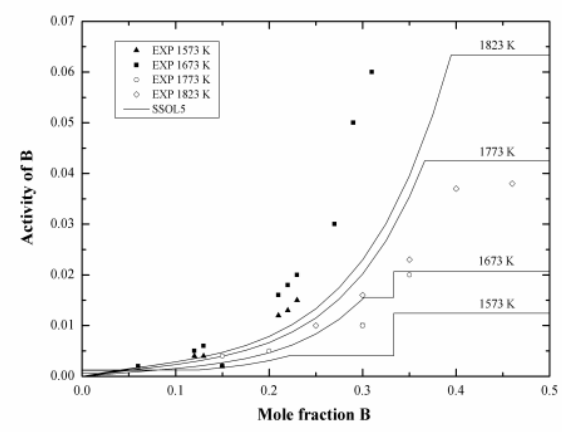

Fig. 10 Activity of B at low boron content in liquid Fe-B alloy calculated at selected temperatures in the SSOL5 database with experimental data [3].

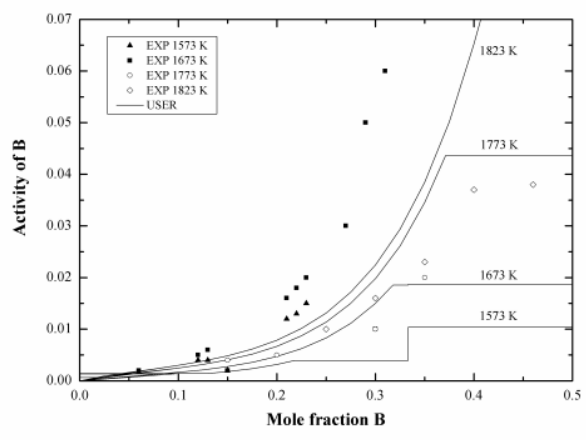

Fig. 11 Activity of B at low boron content in liquid $\mathrm{Fe}-\mathrm{B}$ alloy calculated at selected temperatures in the USER database with experimental data [3].

\section{CONCLUSION}

The assessment of the Fe-B binary system, including calculation of phase diagrams and determining thermodynamic parameters, was carried out in the Thermo-Calc software ver. 4.1. Two databases were used: the commercial database for solid solutions SSOL5 (substitutional approach) and the USER database (intersticial approach). The assessments were compared each other and to experimental data collected by Van Ende et al. Each of the database has some advantages and disadvantages, depending on the property calculated. Some differences are visible only in specific composition ranges. In addition, the experimental data is spread and inaccurate what hinders the evaluation. The following conclusions, referring to the Thermo-Calc calculations, could be drawn:

I. Phase diagram optimization might be in contrary to assessment of thermodynamic properties. One must improve both the shape of the curve (e.g. transformation temperatures) and the thermodynamic functions (Gibbs free energy, enthalpy of formation and heat capacity).

II. Instead of creating new databases it might be beneficial to modify current databases by amending selected parameters.

III. Reference states should be established in order to allow for unmistakable comparison between models and databases.

\section{ACKNOWLEDGEMENTS}

The authors gratefully acknowledge the founding of research fellowship provided by the KMM-VIN community. The present work was supported by Slovak Grant Agency (VEGA) under the project No.2/0153/15.

\section{REFERENCES}

[1]. B. Hallemans, P. Wollants, J.R. Roos, Thermodynamic re-assessment and calculation of the Fe-B phase diagram, $Z$ Metallkde, 85, 1994, 676-682.

[2]. B. Hallemans, P. Wollants, J.R. Roos, Thermodynamic assessment of the Fe-Nd-B phase diagram, Journal of Phase Equilibria, 16(2), 137-149.

[3]. M.A. Van Ende, I.H. Jung, Critical thermodynamic evaluation and optimization of the Fe-B, Fe-Nd, B-Nd and Nd-Fe-B systems, Journal of Alloys and Compounds, 548, 2013, 133-154.

[4]. G.J. Zhou, Y. Luo, Y. Zhou, Thermodynamic Reassessment of the NdFe-B Ternary System, Journal of Electronic Materials, 45(1), 2015, 418-425.

[5]. A. Kroupa, Modelling of phase diagrams and thermodynamic properties using Calphad method - Development of thermodynamic databases, Computational Materials Science, 66, 2013, 3-13.

[6]. V.T. Rompaey, K.C. Hari Kumar, P. Wollants, Thermodynamic optimization of the B-Fe system, Journal of Alloys and Compounds, 8388, 2002, 173-181.

[7]. B. Tummers, DataThief III, 2006. http://datathief.org/. 